\title{
TSA infrared measurements for stress distribution on car elements
}

\author{
Roberto Marsili and Gianluca Rossi \\ Dipartimento di Ingegneria, Università degli Studi di Perugia, Perugia, Via Duranti, no. 1, 06125, Italy \\ Correspondence to: Roberto Marsili (roberto.marsili@unipg.it)
}

Received: 17 May 2017 - Revised: 21 September 2017 - Accepted: 22 September 2017 - Published: 9 November 2017

\begin{abstract}
Because of the continuous evolution of the market in terms of quality and performance, the car production industry is being subjected to more and more pressing technological challenges. In this framework the use of an advanced measurement technique such as thermoelasticity allows the engineers to have a fast and reliable tool for experimental investigation, optimization and validation of the finite element method (FEM) of those critical parts, such as parts of car-frame tables (Marsili and Garinei, 2013; Ju et al., 1997). In this work it is shown how the thermoelastic measurement technique can be used to optimize a Ferrari car frame, as a method of experimental investigation and as a technique of validation of numerical models.

The measurement technique developed for this purpose is described together with the calibration method used in the test benches normally used for fatigue testing and qualification of this car's components. The results obtained show a very good agreement with FEM models and also the possibility of experimentally identifying the concentration levels of stress in critical parts with a very high spatial resolution and testing the effective geometry and material structure.
\end{abstract}

\section{Introduction}

In this work, in order to characterize a car frame, we propose a new measurement technique, and we have designed and realized a test bench to reproduce the real conditions of the use of the car.

The hydraulic shakers furnish the frames with the necessary solicitations to reproduce, in a few hours, the forces that the car will receive during its life.

The complex frame structure and the presence of notches and of weldings cause sudden fatigue fractures for the strain concentrations not always foreseen by the FEM model (Tomlinson and John, 2015; Brouckaert et al., 2012; Becchetti et al., 2010).

Moreover, for the safety coefficient growth, it is not possible to increase the material sections indiscriminately. In this way, in fact, the car would be heavier and its performance on the road would decrease.

Commonly, the mechanical characterization of the car frame is realized in two steps: the first one concerns the development of numerical models. In order to test the numerical solution and to increase the calculation speed, differ- ent types of solvers have been used, in linear and non-linear fields. The second step regards their experimental validation by using strain gauge techniques and accelerometers. Unfortunately this technique furnishes local information only on discrete points. Moreover, the measurement volume depends on the strain gauge dimensions, and often it is not lower than $1 \mathrm{~mm}$ (D'Emilia et al., 2015; Speranzini et al., 2016).

The thermoelastic measurement technique has been used to validate the FEM models in terms of stress distribution. The advantage of this technique is to determine, on the experimental bench, in very low time, the qualitative and quantitative stress distribution on all the car frames.

\section{Thermoelastic theory}

The phenomenon of material changing temperature when it is stretched was first noted by Ghough in 1805, who performed some simple experiments using a strand of rubber, but the first observation in metals of what is now known as the thermoelastic effect was made by Weber in 1830: he noted that a sudden change in tension applied to a vibrating 

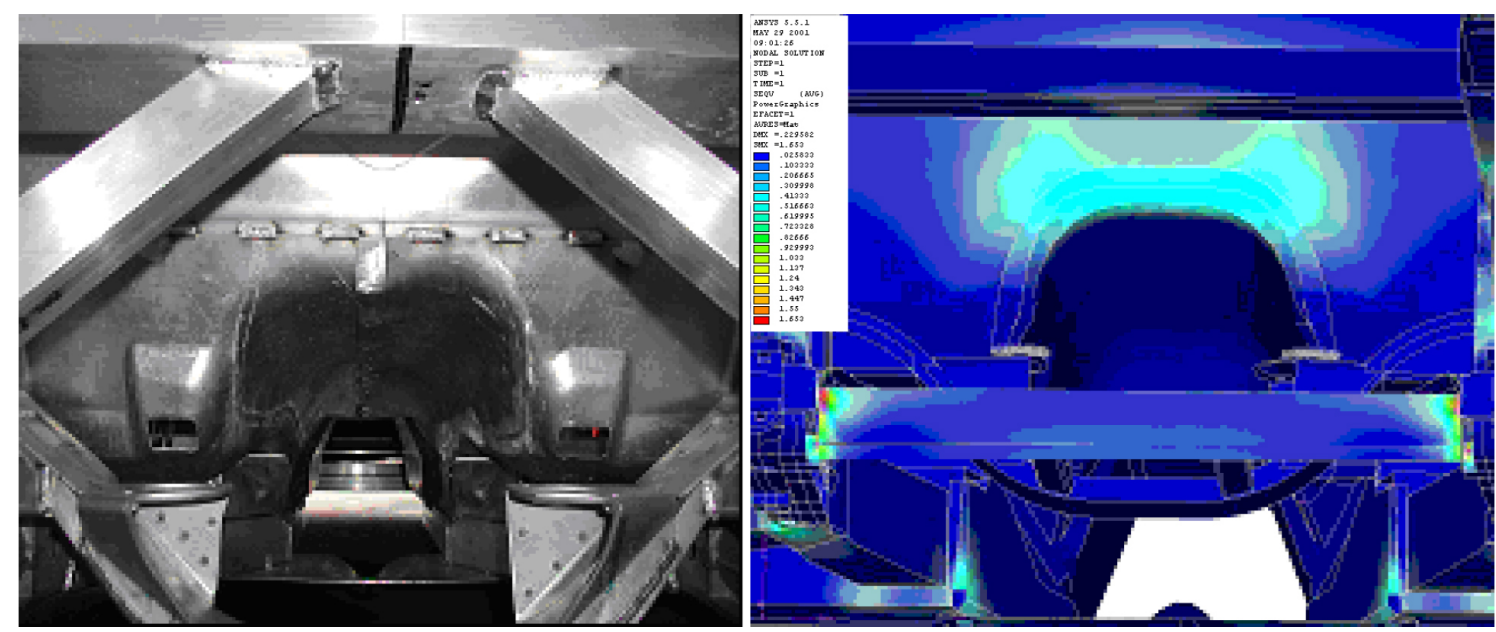

Figure 1. Car frame under analysis and FEM analysis results.
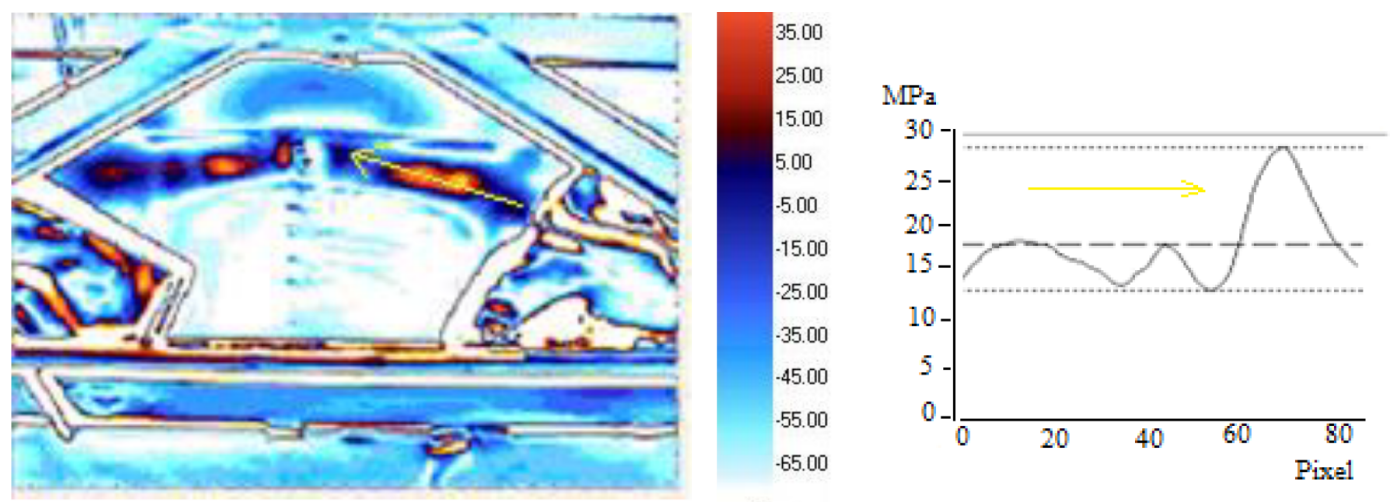

Mpa

Figure 2. Typical thermoelastic result.

wire did not cause the fundamental frequency of the wire to change as suddenly as he expected, but that the change took place in a more gradual fashion. He reasoned that this transitory effect was due to a temporary change in temperature of the wire as the higher stress was applied. In 1974, the Admiralty Research Establishment approached Sira Ltd to determine the relationship between stress and the temperature changes that may be produced by an applied load. Sira confirmed the feasibility and, over the next 4 years, with funding from the English Ministry of Defence, developed a laboratory prototype called Spate (Stress Pattern Analysis by measurement of Thermal Emissions) for applied research. The scientific development of the thermoelastic effect, which is well known on gases, where a temperature variation gives a pressure variation, has been known in solid materials for a short time because of the small variation of temperature induced (in the steel where the stress level is near the yield point, the temperature increases by $0.2{ }^{\circ} \mathrm{C}$ ). The thermoelastic technique for the measurement of stress distribution was developed as soon as they discovered a new temperature measurement technique, based on the emission of infrared radiation, with high sensitivity. The system consists of a differential thermocamera and of software for the post-processing of the image. The thermocamera measures the small temperature variation in the mechanical component induced by a dynamic applied load. Thanks to the software it is possible to have the map of stress distribution on the surface of the structure. The resolutions (supplied from the thermoelastic measurement systems) depend on the material characteristics; they are typically $1 \mathrm{MPa}$ for steel and $0.4 \mathrm{MPa}$ for aluminium. The structure must dynamically be loaded with frequencies sufficiently high so that the thermodynamic conditions in the material can be considered adiabatic. Under these hypotheses it is possible to have a relationship between the mechanical energy and the thermal energy of the structure. The minimum frequency of the applied load depends on the thermal characteristics of the material and on the gradient of the stress fields. The relationship to determine the temperature variation $\Delta T$ of a homogeneous, isotropic, linear elastic 
material with his first stress invariant $\Delta\left(\sigma_{1}+\sigma_{2}\right)$ is

$\Delta T=\frac{T \times \alpha \times \Delta\left(\sigma_{1}+\sigma_{2}\right)}{c_{\mathrm{p}} \times \rho}$,

where $\alpha$ is the thermal expansion coefficient, $T$ is the absolute temperature of the component, $\rho$ is the density, $c_{\mathrm{p}}$ is the thermal capacity at constant pressure, and $\left(\sigma_{1}+\sigma_{2}\right)$ are the components of the tension tensor (Marsili and Garinei, 2012; Harish et al., 2000; Marsili et al., 2008). By the thermoelastic technique it is also possible to measure the map of stress distribution in complex geometries (Marsili and Garinei, 2014a; Dulieu-Barton and Stanley, 1999; Lin and Rowlands, 1995). Normally it is necessary to paint the surface of the mechanical component to increase and make uniform the emissivity. This no-contact technique can have a high spatial resolution, which depends on the optical lenses of the thermocamera.

In order to obtain the stress distribution in terms of quantitative values, a calibration process is required.

This latter can be realized by using a common strain gauge, placed in a zone where the stress gradient is the smallest possible.

In this case the calibration factor $K$ is calculated using the following equation:

$K=\frac{E \cdot\left(\varepsilon_{x}+\varepsilon_{y}\right)}{S_{\text {Avg. }}(1-v)}$.

$E=$ Young's module; $\varepsilon_{x}, \varepsilon_{y}=$ principal strain; $S_{\text {Avg. }}=$ mean grey level of the infrared thermal imaging; $v=$ Poisson's module.

Generally, in order to perform the calibration, a doubleaxis strain gauge is used, with the aim of acquiring the sum of the two principal strains.

\section{Ferrari car frame analysis}

In this work we have analysed the mechanical behaviour of some components of a Ferrari car frame, which has proven critical in the experimental road tests. Figure 1 shows the photo of the frame under study and the FEM model developed. The presence of notches, of weldings and of brazing causes strain concentrations, as highlighted in the numerical analysis (Marsili et al., 2005; Marsili and Garinei, 2014a, b).

On these more stressed points, the use of the classical measurement techniques based on strain gauge or magnetic test are very difficult because of the non-planarity of the surface, the insufficient superficial finish and the small dimensions (Cardelli et al., 2015). The use of thermoelasticity, a measurement technique without contact for the surface distribution of solicitations, provides very important information (Marsili et al., 2009).

The test bench, equipped with the hydraulic shaker, generates a cyclical load on the frame which has been painted with a dull black paint, in order to make uniform the thermal emissivity of the body (Grigg et al., 2000; Lesniak et al., 2013; Offermann et al., 1997; Speranzini et al., 2014).

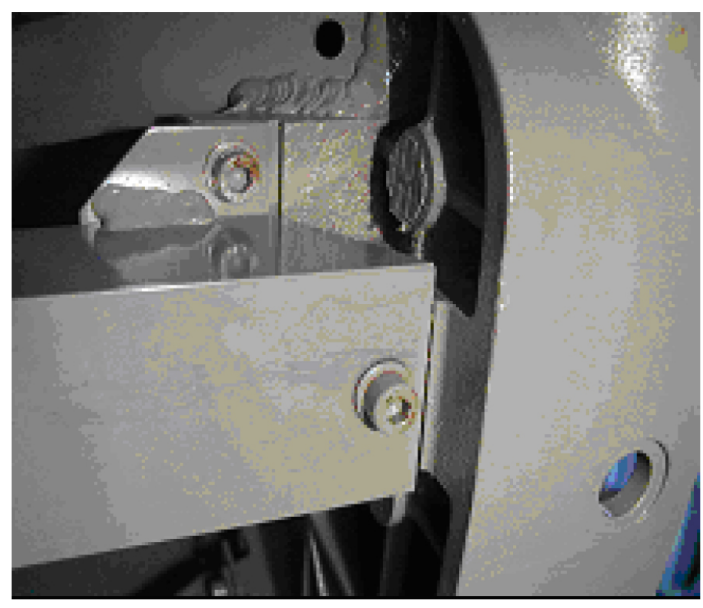

Figure 3. A car bracing analysis.

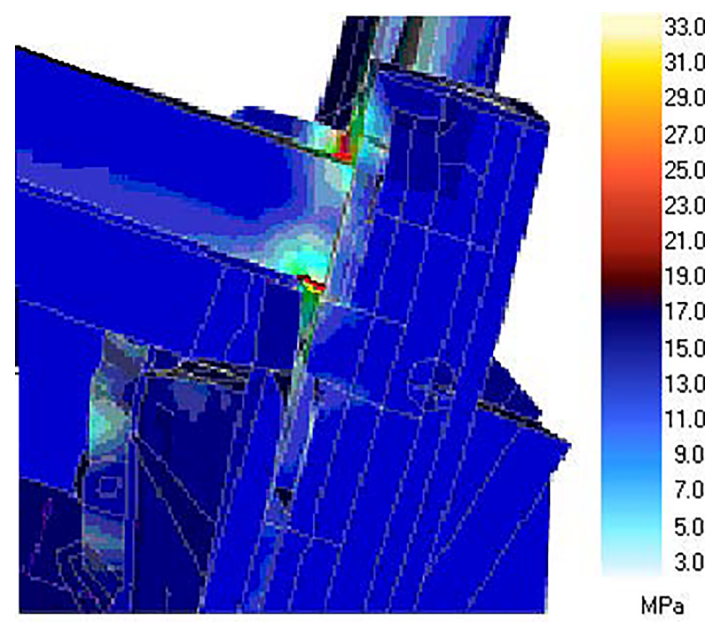

Figure 4. Typical FEM result.

An electrical strain gauge has been pasted on the frame to convert the thermographic frame into a stress frame, as well as to generate the reference signal necessary to the TSA system, to synchronize the frame grabber with the dynamic cycle load.

In fact the Delta Therm 1550 uses the lock-in amplifier technique to acquire only the temperature change synchronous with the applied load.

At the same time it allows us to improve the signal-noise ratio. Figure 2 presents a typical result that can be obtained using this type of measurement technique. In the same picture it is also possible to determine the stress concentrations near the constraint section and the zones where the shut is present. The thermoelastic stress map has been scaled by means of a calibration factor $K$, calculated with Eq. (2), using the strain gauge. In this way, through Eq. (1) a differential thermographic frame becomes a stress distribution map.

The previous TSA image is very useful for validating the FEM distributions in terms of the sum of principal stresses. 


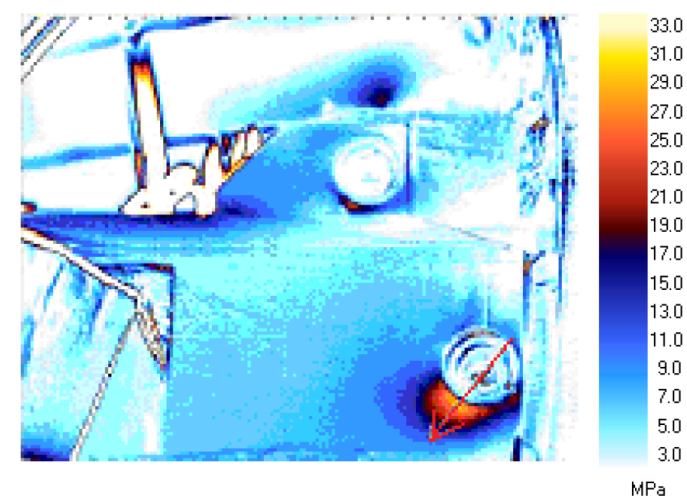

Figure 5. Thermoelastic stress analysis.

In this case the correlation between FEM analysis and experimental results appears clearly. Drawing an interrogation line as shown in Fig. 2, it is possible to evaluate the stress trend along the same line as reported to the right of the same Fig. 2. From the analysis of two typical interrogation lines, the gap between FEM and thermoelastic analysis is evaluated. The maximum difference found is $3 \mathrm{MPa}$. The qualitative and quantitative coincidence of the experimental and numerical results now allows the use of the model to change the geometry and the sections of the frame, or to insert a bracing, in order to reduce at the maximum the concentration of strains, without repeating the experimental tests, with economic and time advantages.

Figure 3 shows an example of a bracing welded on the frame. By the numerical analysis it is possible to see the strain concentration in correspondence to the bracing that could cause fatigue breaks of the component.

The same strain concentration is also seen in the experimental analyses by the thermoelastic system (Fig. 5). The thermoelastic experimental analysis highlights an elevated concentration of strains also around the screw not predicted, instead, by the FEM analysis.

\section{Experimental calibration and uncertainty analysis}

Normally in the thermoelastic measurement the calibration is based on the measurement of the deformation by a strain gauge rosette in a point of the structure. To put on the strain gauge rosette we have considered certain points with a high and regularly distributed solicitation. Repeated measurements by stain gauge of the principal strains and the relative measure of the infrared intensity radiation allow us to estimate the calibration factor $K$, varying the applied load, the observation area and the excitation frequency. The best available estimate of the thermoelastic constant $k$ value is $k=0.14 \mathrm{MPa} \mathrm{mV}^{-1}$. The experimental standard deviation is $s(k)=0.01 \mathrm{MPa} \mathrm{mV}^{-1}$.

The composed uncertainty in the value of the thermoelastic constant $K$ can be determined based on a relationship

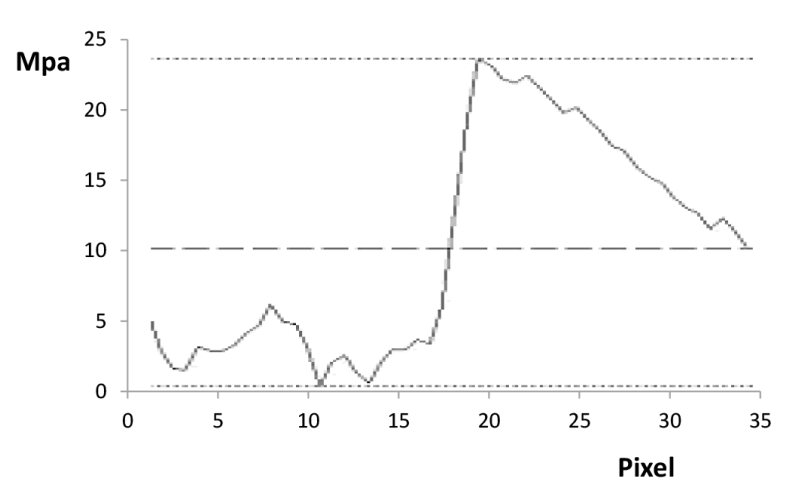

(Eq. 2) as follows (Eq. 3):

$$
\begin{aligned}
\partial K & =\left|\frac{\partial K(E)}{\partial E} \partial E\right|+\left|\frac{\partial K\left(\varepsilon_{x}\right)}{\partial \varepsilon_{x}} \partial \varepsilon_{x}\right|+\left|\frac{\partial K\left(\varepsilon_{y}\right)}{\partial \varepsilon_{y}} \partial \varepsilon_{y}\right| \\
& +\left|\frac{\partial K(V)}{\partial V} \partial V\right|+\left|\frac{\partial K(v)}{\partial v} \partial \nu\right| .
\end{aligned}
$$

Assuming a relative uncertainty of $2 \%$ in the Poisson and Young moduli of the materials, of $2 \%$ in the determination of the principal strain $\varepsilon$ and $2 \%$ in the rms of the signal measured by the infrared sensor $V$, the combined standard uncertainty is $\delta K=0.009$, and so the relative uncertainty is

$\frac{\partial K}{K}=9 \%$.

\section{Conclusions}

In this work a Ferrari car frame has been characterized from the mechanical point of view to single out the areas with higher concentrations of stress. Firstly, a model of numerical simulation has been validated using classic measurement techniques based on the use of a strain gauge and through thermoelastic techniques.

This last analysis has confirmed the results obtained from the numerical point of view and in certain cases we have identified areas with tension concentrations not foreseen with the FEM analysis.

The use of a strain gauge, as an instrument of reference, has given us the possibility of calculating the calibration constant and estimating the measurement uncertainty.

Data availability. No data sets were used in this article.

Competing interests. The authors declare that they have no conflict of interest.

Edited by: Rosario Morello

Reviewed by: two anonymous referees 


\section{References}

Becchetti, M., Flori, R., Marsili, R., and Moretti, M.: Comparison between digital image correlation and thermoelasticity for strain field analysis, AIP Conf. Proc., 1253, 233-240, https://doi.org/10.1063/1.3455462, 2010.

Brouckaert, J. F., Marsili, R., and Rossi, G.: Development and experimental characterization of a new non contact sensor for blade tip timing, 10th International Conference on Vibration Measurements by Laser and Noncontact Techniques - AIVELA, Ancona, ITALY, 27-29 June 2012 Conference Proceedings, 1457, 61-68, https://doi.org/10.1063/1.4730543, IDS Number: BBI34 ISSN: 0094-243X ISBN: 978-0-7354-1059-6, 2012.

Cardelli, E., Faba, A., Marsili, R., Rossi, G., and Tomassini, R.: Magnetic nondestructive testing of rotor blade tips, J. Appl. Phys., 117, 17A705, https://doi.org/10.1063/1.4907180, 2015.

D'Emilia, G., Gaspari, A., and Natale, E.: Dynamic Calibration Uncertainty of Three-Axis Low Frequency Accelerometers: Test Rig and Procedure Aspects, ACTA IMEKO, December, 4, 7581, 2015.

Dulieu-Barton, J. M. and Stanley, P.: Applications of thermoelastic stress analysis to composite materials, Strain, May, 41-48, 1999.

Grigg, J. S., Dulieu-Barton, J. M., and Shenoi, R. A.: Validation of a complex composite construction using thermoelastic stress analysis, Proceedings of the SEM IX International Congress on Exp. Mech., 5-8 June, Orlando, Florida, 355-358, 2000.

Harish, G., Szolwinski, M. P., Farris, T. N., and Sakagami, T.: Evaluation of fretting stress through full-field temperature measurement, ASTM STP, 2000.

Ju, S. H., Lesniak, J. R., and Sandor, B. I.: Numerical simulation of stress intensity factors via the thermoelastic technique, Exp. Mech., 37, 278-284, 1997.

Lesniak, J. R. and Bradley, R. B.: A High-Speed Differential Thermographic Camera, Stress Photonics Inc., 565 Science Drive Madison, WI 53711, 2013.

Lin, S. T. and Rowlands, R. E.: Thermoelastic Stress Analysis of Orthotropic Materials, Exp. Mech., September, 257-265, 1995.

Marsili, R. and Garinei, A.: A new diagnostic technique for ball screw actuators, Measurement, Elsevier Science, 45, 819-828, https://doi.org/10.1016/j.measurement.2012.02.23, 2012.

Marsili, R. and Garinei, A.: Design of an optical measurement system for dynamic testing of electrospindles, Measurement, 46, 1715-1721, https://doi.org/10.1016/j.measurement.2013.01.006, 2013.
Marsili, R. and Garinei, A.: Thermoelastic Stress Analysis of the Contact Between a Flat Plate and a Cylinder, Measurement, Journal of the International Measurement Confederation, 52, 102110, https://doi.org/10.1016/j.measurement.2014.03.005, 2014a.

Marsili, R. and Garinei, A.: Development of a new capacitive matrix for a steering wheel's pressure distribution measurement, Int. J. Ind. Ergonom., 44, 114-119, https://doi.org/10.1016/j.ergon.2013.11.012, 2014b.

Marsili, R., Brustenga, G., Moretti, M., Pirisinu, J., and Rossi, G.: Measurement on rotating mechanical component by thermoelasticity, Journal Applied Mechanics and Materials, ISBN 0-87849987-3 ISSN 1660-9336, 3-4, 337-342, 411-416, SCOPUS: 2s2.0-33745170726, 2005.

Marsili, R., Moretti, M., and Rossi, G. L.: Thermoelastic Modal Stress Analysis, IMAC XXVI Conference \& Exposition on Structural Dynamic, Orlando, Florida USA, 4-7 February 2008, ISBN: 0912053984 ISBN 9781605600666 , SCOPUS: 2-s2.084861536363, 2008.

Marsili, R., Rossi, G., Becchetti, M., and Flori, R.: Stress and strain measurements by image correlation and Thermoelasticity, Society for Exp. Mech. - SEM Annual Conference and Exposition on Experimental and Applied Mechanics 2009, Volume 1, Pages 70-75, Albuquerque, NM, United States, 1-4 June 2009, Code 78716, ISBN: 978-161567189-2, 978-161567189-2, SCOPUS: 2-s2.0-73349141710, 2009.

Offermann, S., Beaudoin, J. L., Bissieux, C., and Frick, H.: Thermoelastic Stress Analysis Under Non-adiabatic Conditions, Exp. Mech., 37, 409-413, 1997.

Tomlinson, A. and John, R.: An investigation of crack closure using thermoelasticity, Rachel A, University of Sheffield UK, 2015.

Speranzini, E. and Agnetti, S.: The technique of digital image correlation (DIC) to identify defects in glass structures, Struct. Control. Hlth., 21, 2015-2029, https://doi.org/10.1002/stc.1629, 2014.

Speranzini, E., Agnetti, S., and Corradi, M.: Experimental analysis of adhesion phenomena in fibre-reinforced glass structures, Composites part B, 101, 155-166, https://doi.org/10.1016/j.compositesb.2016.06.074, 2016. 